\title{
Preface: analytical models for financial modeling and risk management
}

\author{
Constantin Zopounidis $^{1,2} \cdot$ Michalis Doumpos $^{1} \cdot$ \\ Kyriaki Kosmidou ${ }^{3}$
}

Published online: 17 May 2018

(C) Springer Science+Business Media, LLC, part of Springer Nature 2018

Banks, financial institutions, and companies have developed financial modeling worldwide, and risk management techniques and processes have been adopted by businesses to implement risk-based policies and practices. Financial modeling and risk management are of great interest since they combine the principles of fundamental science and real-world applications.

The level of sophistication of financial modeling tools, techniques, and approaches has increased considerably over the past couple of decades, driven by the experiences gained from responding to turmoil and crises, the strengthening of regulatory requirements, the adoption of new technological advances, and the introduction of new financial products and instruments. Analytical techniques are of paramount importance in financial modeling and decision-making. Operations research/management science and other related analytical disciplines are highly relevant to this area, providing the means for normative, descriptive, prescriptive, and predictive modeling.

This special volume, was prepared on the occasion of the 6th International Conference of the Financial Engineering and Banking Society, which was held in Malaga, Spain, during 10-12 June 2016. It explores recent advances in the use of analytical models in all areas of

Michalis Doumpos

mdoumpos@dpem.tuc.gr

Constantin Zopounidis

kostas@dpem.tuc.gr

Kyriaki Kosmidou

kosmid@econ.auth.gr

1 Financial Engineering Laboratory, School of Production Engineering and Management, Technical University of Crete, University Campus, 73100 Chania, Greece

2 Audencia Business School, Institute of Finance, 8 route de la Jonelière, B.P. 31222, 44312 Nantes Cedex 3, France

3 Division of Business Administration, Department of Economics, Aristotle University of Thessaloniki, 54124 Thessaloniki, Greece 
financial modeling and risk management, covering both new theoretical developments and new empirical results.

Extended versions of papers presented at the conference as well as other submissions were submitted to the special volume. Of 53 papers, 22 were accepted after a rigorous review process. These cover a wide range of topics, including derivatives, asset pricing, risk management, financial and banking institutions, portfolio selection, and fund management, among others.

The volume starts with the paper of Consiglio, Lotfi, and Zenios, who present portfolio models for sovereign credit default swaps (CDS) to diversify idiosyncratic risk. Different investment strategies are considered for long investors, speculators, and hedgers, under a bi-objective scheme based on return and conditional value at risk (CVaR). The models are tested on data from Europe and they are found superior when compared to an established CDS index.

In the second paper, Blomvall and Ekblom develop a stochastic programming framework for hedging currency and interest rate risk. Their approach considers skewness and kurtosis in exchange rates, transaction costs, systematic risks, as well as hedging costs (through the term premia). Optimal hedging strategies are constructed using three criteria-variance, expected shortfall, and profits. The results provide insights into the role of the chosen performance objectives.

Next, Gubareva and Borges develop an approach based on derivatives to assess the economic capital requirements for interest rate and credit risk in banking institutions. Time-series of CDS and interest rate swaps rates are used as proxy measures of risk. The proposed framework is applied to analyze risk dynamics of sovereign debt of 30 developing countries.

The next two papers focus on derivatives pricing. In the first paper, Sierag and Hanzon provide a generalization of the binomial tree model based on the Pascal's simplex, which allows the pricing of multiple assets. They present the theoretical aspects of the proposed model, illustrate it through numerical examples, and compare the results against other similar models.

The second paper in this part is by Funahashi and Higuchi, who present the derivation of an approximation formula for a single barrier option under local volatility models, stochastic volatility models, and their hybrids. The accuracy of the proposed approach is analyzed through numerical examples.

Karouzakis, Hatgioannides, and Andriosopoulos present a novel approach for pricing constant maturing swaps (CMS). Their model relaxes the common assumption of flat term structure, allowing the handling of more realistic cases. The model is calibrated to market data for Euro Money Market Instruments and the obtained results are found to be superior when compared to other standard models.

The next six papers involve portfolio selection, optimization, and management. This series starts with the paper of Kim, Kim, and Fabozzi, who provide an overview of recent advances in robust optimization for investment management. Robust optimization has become a popular approach for modeling uncertainty, not only in portfolio selection problems, but also in other areas of financial decision making. The overview covers such areas as asset allocation, portfolio selection, and derivatives.

Kapsos, Christofides, and Rustem introduce robust risk budgeting, a robust variant of standard risk budgeting that deals with the uncertainty in the input parameters. Their paper proposes a computationally efficient formulation of the problem as well as a framework for incorporating the uncertainty associated with the covariance matrix, without adding complexity to the problem. Empirical results from data involving exchange traded funds (ETFs) are presented to demonstrate the performance of the proposed approach. 
Paç and Pınar also consider a robust optimization approach for portfolio selection. They consider an investment environment combining risky and risk-free assets and analyze the performance of naïve diversification. Using an analytic approach, it is shown that as uncertainty in asset returns increases, the optimal portfolio converges to a naïve diversification strategy. Decision rules for optimal portfolio selection are derived under in a CVaR context.

Kremer, Talmaciu, and Paterlini present and extensive empirical analysis of eight state-ofthe-art risk-minimization schemes for risk factor-based portfolio construction. The analysis considers 25 risk factors using data from five regions. The results show that each factor yields positive premia in exchange of risk, whereas multi-factor portfolios from different regions reduce volatility and extreme risk measures.

Kim, Kim, Kim, and Fabozzi present a comprehensive analysis on robust portfolio performance for equity portfolios constructed in the U.S. market during the period 1980-2014. The results confirm the advantage of robust portfolio optimization for controlling uncertainty while efficiently allocating investments.

Kalyagin, Koldanov, Koldanov, and Pardalos present a network-based approach for stock market analysis and the identification of similarities in assets' returns. The model is based on a market graph, and an optimal statistical procedure is proposed for market graph identification in a sign similarity network. The proposed procedure is not sensitive to the assumptions on the distribution of stock attributes, thus overcoming the limitations of Pearson correlation networks, which assume multivariate normality.

In the next paper, Temocin, Korn, and Selcuk-Kestel consider the optimal portfolio problem with minimum guarantee protection in a defined contribution pension scheme. Two strategies are compared in a random and deterministic setting. Optimal strategies are constructed using a stochastic control approach. The results show that both strategies can be preferred according to participants' expectations.

Giuzio, Eichhorn-Schott, Paterlini, and Weber present an approach based on constrained regression models using regularization methods for hedge fund index replication. A new model is introduced based on a log-penalty criterion, and it is shown to outperform a standard LASSO regression approach. Extensive empirical results are provided for a set of 28 hedge fund indices.

This part of the special volume involving portfolio and fund management closes with the paper by Nicolosi, Angelini, and Herzel, who examine the problem of a fund manager whose compensation depends on relative performance with respect to a benchmark index. A dynamic market setting is considered, combining risk assets with a money market account, and an optimal solution is found using the martingale approach for a manger that optimizes the expected utility of the final wealth with a nonconcave objective function.

The next three papers present empirical studies on the CDS and stock markets. Chalamandaris and Vlachogiannakis propose a combination of LASSO with panel-consistent estimation methods to investigate whether financial ratios are used in the decision-making process of CDS traders. The obtained results show that financial statement information does play a role for proactive analysts, news traders, as well as value traders, who respond asymmetrically to financial ratio updates of different sign and intensity.

Koutmos studies the dynamic interdependencies between CDS spread among several European Union countries during the period between October 2004 and July 2016. The empirical findings show that the interdependencies between countries' credit risks are heterogeneous when considering the 2008-2009 financial crisis and 2011-2013 European debt crisis.

The third paper in this series, by Cano-Berlanga and Giménez-Gómez, analyzes the evolution of the Chinese stock markets through a Markov-Switching asymmetric GARCH in four 
different time frames. The analysis considers the effect of legal reforms, differences between the main Chinese markets, as well as sentiment effects.

In the next paper, Jiang, Wang, Wang, and Ding propose a default prediction method for peer-to-peer (P2P) lending. In addition to standard "hard" data often used for credit scoring, the proposed approach also considers soft information derived from the description of the loans in order to construct relevant features for default prediction. An empirical analysis using real-word data from a major P2P lending platform in China shows that the proposed method can provide better loan default predictions than existing methods based only on hard information.

The next two paper present applications of data envelopment analysis (DEA) in banking. Tziogkidis, Matthews, and Philippas examine the effects on the productivity of Greek commercial banks of sector regulatory reforms in the pre-Euro era, using the Global Malmquist Index. The analysis is based on a bootstrap approach, which uses the Pearson system random number generator instead of kernel density estimators.

The paper of Ouenniche and Carrales focuses on UK commercial banks. The authors proposed an approach that combines DEA with regression analysis. The latter provides information about the relevance of the chosen inputs and outputs. The adopted DEA models allow the consideration of both inputs and outputs, only inputs, or only outputs. The results show that standard DEA models tend to over- or under-estimate efficiency scores depending on the orientation of the analyses. However, the addition of regression analysis proves effective in improving discrimination in efficiency estimates for banks.

The special volume closes with the paper of Galariotis, Germain, and Zopounidis. The authors present the application of a multicriteria evaluation approach to the assessment of the performance of football clubs in France. The assessment combines financial, business, and sports attributes. At a second stage, a partial least squares structural equation modeling approach is used to examine the relationships between the above performance dimensions.

In closing, we express our sincere thanks to all authors whose contributions have been essential to this rich and high-quality special volume. We also acknowledge the support of all reviewers who devoted considerable time to provide critical evaluations, insightful comments, and constructive suggestions for the submitted papers. Without their help it would have been impossible to achieve this volume's high standards. 